\title{
Diagnostic strategies for suspected pulmonary embolism
}

\author{
A.B. Donkers-van Rossum
}

Diagnostic strategies for suspected pulmonary embolism. A.B. Donkers-van Rossum. C) ERS Journals Ltd 2001.

ABSTRACT: Pulmonary embolism often remains a difficult diagnosis for the clinician, particularly in patients with comorbidity factors. This is in contrast with the availability of effective treatment, which should be prescribed as soon as possible.

To date, there is still no ideal diagnostic test that is accurate, safe, readily available and cost-effective.

Recent technical advances in computed tomography, magnetic resonance imaging and laboratory findings have raised new possibilities in the diagnosis of pulmonary embolism.

This review covers the performance of different diagnostic tests, and focuses on the advantages and limitations of single diagnostic tests and the clinical usefulness of these tests in diagnostic strategies.

Eur Respir J 2001; 18: 589-597.
Correspondence: A.B. Donkers-van Rossum, Dept of Radiology, Catharina Hospital Eindhoven, PO Box 1350, Eindhoven $5602 \mathrm{AZ}$, the Netherlands. Fax: 31413212348

Keywords: Computed tomography magnetic resonance imaging pulmonary embolism radiology

Received: October 122000 Accepted after revision May 312001
Diagnosis of pulmonary embolism (PE) remains a major clinical problem. The annual incidence of clinically suspected $\mathrm{PE}$ is estimated to be 2-3 per 1,000 inhabitants in the western world $[1,2]$. Of these patients, only approximately one-third will actually have a pulmonary embolus [3-5]. Treatment of patients with $\mathrm{PE}$ is indicated, because the mortality of $\mathrm{PE}$ without treatment is $\sim 25 \%$ [6]. Conversely, treatment of all patients will lead to unnecessary treatment in two-thirds of patients, with possible haemorrhagic complications. Hence, an accurate diagnosis is warranted to prevent unnecessary mortality and morbidity.

Unfortunately, the clinical signs and symptoms alone of PE are nonspecific. The diagnosis of PE therefore relies heavily on additional tests, including imaging techniques. There have been major developments regarding these diagnostic tests in recent years, implying new diagnostic strategies for acute PE and raising new opportunities in the diagnosis of PE. However, the diagnosis of PE remains problematical and continues to challenge the skills of both the physician and diagnostic imager.

It is the intention of this paper to give an overview of the presently available diagnostic tests, with their advantages and disadvantages, and the clinical usefulness of these tests in diagnostic strategies.

\section{Diagnostic tests}

\section{Clinical assessment}

Although the signs and symptoms of PE are nonspecific, there are some "classical" clinical signs, which raise the suspicion of PE. Circulatory collapse caused by massive PE presents as shock or syncope. It is sometimes accompanied by severe dyspnoea and chest pain and there may be signs of acute right heart failure. Acute breathlessness, often with tachycardia, may be the result of a submassive embolus. There may be chest pain, tachypnoea and cyanosis. Smaller emboli may cause pleuretic chest pain, with or without breathlessness and haemoptysis.

According to the clinical presentation, together with electrocardiography, chest radiography and laboratory findings, the physician can make a clinical pretest probability of PE. Some studies have demonstrated that the clinical pretest probability may be a useful adjunct to further diagnostic tests. In the PIOPED study [5], a high clinical probability combined with a

Previous articles in this series: No. 1: Ghaye B, Dondelinger RF. Imaging guided thoracic interventions. Eur Respir J 2001; 17: 507-528. No. 2: Vansteenkiste JF, Stroobants SG. The role of positron emission tomography with ${ }^{18}$ F-fluoro-2-deoxy-D-glucose in respiratory oncology. Eur Respir J 2001; 17: 802-820. No. 3: Kauczor HU, Chen XJ, van Beek EJR, Schreiber WG. Pulmonary ventilation imaged by magnetic resonance: at the doorstep of clinical application. Eur Respir J 2001; 17: 1008-1023. No. 4: Hansell DM. Small airways diseases: detection and insights with computed tomography. Eur Respir $J$ 2001; 17: 1294-1313. No. 5: Franquet T. Imaging of pneumonia: trends and algorithms. Eur Respir J 2001; 18: 196-208. No. 6: Ferretti GR, Bricault I, Coulomb M. Virtual tools for imaging of the thorax. Eur Respir J 2001 ; 18: $381-392$. 
high probability ventilation/perfusion $\left(V^{\prime} / Q^{\prime}\right)$ lung scan, had a $96 \%$ positive predictive value for $\mathrm{PE}$, whereas a low clinical probability combined with a normal/near normal or low probability $V^{\prime} / Q^{\prime}$ lung scan had a 97\% negative predictive value for PE. However, some $75 \%$ of the patients in the PIOPED study did not fit into the aforementioned categories. Thus, combining the clinical probability with the $V^{\prime} /$ $Q^{\prime}$ lung scan in this study improved the predictive accuracy only for a minority of patients.

Results of a study by MinIATI et al. [7] where clinical probability was combined with the perfusion lung scan indicated that: 1) a high or indeterminate clinical probability combined with an abnormal $V^{\prime} / Q^{\prime}$ lung scan, compatible with PE makes the diagnosis of PE very likely; 2) a low clinical probability paired with a $V^{\prime} / Q^{\prime}$ lung scan not be compatible with PE, makes the diagnosis very unlikely; 3) when clinical probability and $V^{\prime} / Q^{\prime}$ lung scan results are discordant, the pretest probability is neither sufficiently high nor sufficiently low to render the diagnosis or exclusion of $\mathrm{PE}$ reasonably certain. Under these circumstances further diagnostic tests are required. In contradiction with the PIOPED findings, most patients in the study of Miniati et al. [7] had concordant clinical and perfusion scan findings. Thus it appears, according to their results, that in most patients, PE could be diagnosed or excluded noninvasively by combining well-defined clinical estimates of PE with the perfusion lung scan findings.

\section{D-dimer}

D-dimer is a breakdown product that is formed when cross-linked fibrin is cleaved by plasmin. Increased levels of plasma D-dimer may be encountered with breakdown of thrombi, although this is not specific. There are other causes (such as neoplasm, sepsis, postoperative state) of fibrinolysis, hence, the specificity of all D-dimer assays for PE is low. The potential value of a D-dimer test lies, therefore, in its ability to exclude PE. And such a test must be of high sensitivity and must have a high negative predictive value.

Plasma D-dimer levels can be measured by a variety of methods. Latex agglutination tests (such as Simply Red and Tinaquant) are simple, fast and inexpensive, but of insufficient sensitivity for clinical use [8, 9]. The enzyme-linked immunosorbent assay (ELISA) methods seemed promising, but required plasma preparation and were labour intensive. More recent improvements have yielded fast and acutely applicable tests such as the new rapid ELISA test $[10,11]$. These are better suited for emergency situations. Sensitivity and negative predictive value varies up to as high as $100 \%[11,12]$. Overall, the ELISA test has the best quoted sensitivities for PE, whereas the latex tests have the poorest. Unfortunately, most studies did not determine the accuracy of $\mathrm{D}$-dimer assays with respect to the gold standard pulmonary angiography. Instead, most studies used noninvasive tests to exclude or include PE. Nevertheless, it is noteworthy that in a large study [11], no patient with a negative D-dimer test had a thromboembolic event at 3-month followup. So, in a patient with a low clinical suspicion of PE and a negative D-dimer test of the rapid ELISA type, PE can be excluded.

It also needs to be noted that most patients with comorbid conditions (such as inpatients) have a higher D-dimer than the cut-off value, thus questioning its usefulness in excluding $\mathrm{PE}$ in hospitalized patients. Miron et al. [13] evaluated the effectiveness of D-dimer measurement in patients suspected of PE occurring during hospital stay. The specificity of D-dimer was only $7 \%$ and they concluded that D-dimer measurement appears to be useless in hospitalized patients.

\section{Ultrasonography}

PE and deep vein thrombosis (DVT) are both entities of one disease, namely venous thromboembolism. It therefore seems logical in the diagnosis of PE, to search for DVT, as it is also suggested that PE may originate from the deep veins. Both disorders require the same treatment, hence, detection of DVT in a patient suspected of $\mathrm{PE}$ thus renders further testing for PE useless, and anticoagulant therapy can be started.

Lower limb venous compression ultrasonography is widely used to detect DVT. With the patient in the supine position, the femoral and popliteal veins are examined with a high Mhz linear transducer. The diagnosis is primarily based on incompressibility of a venous segment. Secondary signs for the diagnosis of DVT are an abnormal or absent flow or visualization of a clot in the vein, but diagnosis could not be established on only these signs; there is a high sensitivity and specificity for ultrasound in the diagnosis of DVT in patients with symptomatic proximal DVT (over 97\%) [14, 15]. The sensitivity of ultrasonography for diagnosis of distal DVT is, however, substantially lower. Therefore, various strategies are based on serial ultrasonography, requiring repetition of the examination over a period of $7-14$ days $[16,17]$ to detect proximal extension of DVT.

In patients suspected for PE and very often asymptomatic for DVT, the sensitivity of ultrasonography drops, with sensitivity and specificity in the order of 35-99\% [18-24]. Whether examination of the lower limb veins should be routinely performed in all patients suspected of PE is another problem. Protagonists of this view argue that, in particular, ultrasonography of the lower limb veins is a low-cost, noninvasive test that is fast and easy to perform [22, 25-27]. Other investigators, however, reason that too few patients with suspected PE have DVT to merit ubiquitous use of ultrasound [21, 28]; the prevalence of DVT in suspected PE is $\sim 18 \%$ and in proven $\mathrm{PE}$ is $36-45 \%$ [29].

\section{Lung scintigraphy}

The usual next step after an initial conventional chest radiograph, still is to perform a $\left(V^{\prime} / Q^{\prime}\right)$ lung scintigram. 
The perfusion scintigram is performed with technetium-99m $\left({ }^{99 \mathrm{~m}} \mathrm{Tc}\right)$-labelled macroaggregated albumin. Images are acquired via a low-energy, large field of view gamma camera, in six projections; anterior, posterior, left and right posterior oblique and left and right lateral projections. In a normal lung scan, the lung margins are concave and uninterrupted. PE characteristically appears as segmental, pleural-based perfusion defects. The timing of the perfusion scan in relation to the onset of symptoms is important, since resolution of the thrombus takes place by activation of thrombolysis. These changes take place over the course of several hours, so the perfusion scan should therefore be performed within $24 \mathrm{~h}$ of the onset of symptoms.

After the perfusion scan, a concurrent ventilation scan is performed, preferably with Krypton- $81 \mathrm{~m}$ $\left({ }^{81 \mathrm{~m}} \mathrm{Kr}\right)$ gas, and the same six views are obtained. The results of the ventilation lung scan can be combined with the perfusion lung scan. If the perfusion scan shows no defects, this is called a normal perfusion lung scan. When there is a perfusion segmental defect and normal ventilation in the same region, this is called a mismatch and is suspect for positive $\mathrm{PE}$, i.e. high-probability $V^{\prime} / Q^{\prime}$ lung scan. If there are both perfusion and ventilation defects in the same segment, this is called a matching defect and makes PE less probable, but does not exclude PE. This type of scan is called a nonhigh probability $V^{\prime} / Q^{\prime}$ lung scan.

There are several categorical criteria for the diagnosis of PE, like the MCNIEL et al. [30], BIELLO et al. [31] or PIOPED-classifications [5], with criteria for very low-, low-, indeterminate-, normal- and highprobability for PE. Unfortunately, there is a large difference in interpretation among expert readers $(25-30 \%)$, especially in the classification of low or indeterminate probability $V^{\prime} / Q^{\prime}$ lung scans [5]. Another more clinically orientated suggestion for a classification was made by Hull et al. [32]. They classified $V^{\prime} / Q^{\prime}$ lung scans as either normal- or highprobability or nondiagnostic, and for daily clinical practice, these modified, revised PIOPED criteria, in the present author's opinion, works best.

A $V^{\prime} / Q^{\prime}$ lung scan is called a high-probability lung scan if there are $\geqslant 2$ perfusion defects $(>75 \%$ of segment, mean $25-27 \%$, defects $<25 \%$ do not count) without a ventilation defect (fig. 1), with a chance of not having $\mathrm{PE}$ of $12 \%[4,5,33]$. The $V^{\prime} / Q^{\prime}$ lung scan is called normal if there are no perfusion defects. This can be used to exclude PE (chance of having PE, $0.2 \%$ [34]). So, a normal perfusion lung scan effectively rules out clinically relevant $\mathrm{PE}$, and anticoagulants can be safely withheld. Unfortunately most patients suspected of PE $(>60 \%)$ will have a so-called nondiagnostic $V^{\prime} / Q^{\prime}$ lung scan, and this nondiagnostic $V^{\prime} / Q^{\prime}$ lung scan neither excludes nor establishes the diagnosis of PE, and, therefore, warrants further testing. The chance of having $\mathrm{PE}$ among these patients varies from $25-30 \%$ [5].

Another question is whether the combination of ventilation and perfusion lung scanning is as valuable as suggested. The ventilation lung scan was added to the perfusion scan to improve it's specificity, but does

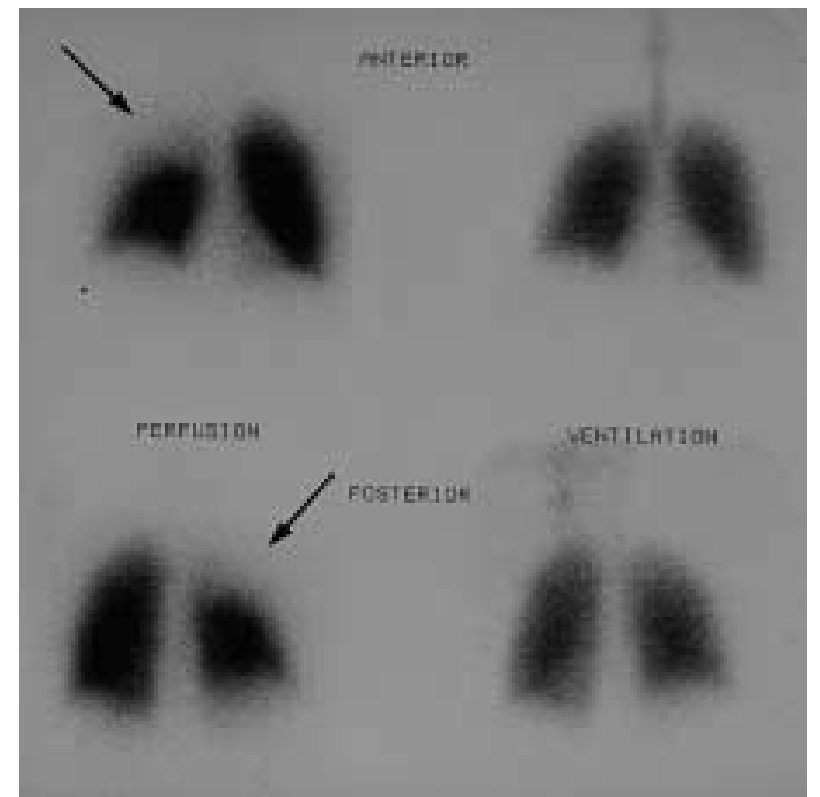

Fig. 1.- An example of a high probability ventilation/perfusion lung scan. There is a perfusion defect in the right upper lobe (arrow) with normal ventilation in this region.

it really improve the diagnostic yield of lung scintigraphy? In the PIOPED study [5], most patients with a high probability $V^{\prime} / Q^{\prime}$ lung scan had PE, but only a minority of patients with angiographically proven $\mathrm{PE}$ had a high-probability $V^{\prime} / Q^{\prime}$ lung scan. Virtually all patients with PE had $V^{\prime} / Q^{\prime}$ lung scans of high-, indeterminate-, and low-probability, but so did most of the patients without PE. Hence, in a sizeable proportion of patients, PE was associated with a $V^{\prime} / Q^{\prime}$ lung scan other then a high probability scan [5]. Because of these findings, Miniati et al. [35], in their study, wanted to reconsider the value of perfusion scanning alone, without ventilation imaging in the diagnostic workup of patients with suspected PE. They showed that the perfusion scan had an excellent sensitivity $(92 \%)$ and that accurate diagnosis of $\mathrm{PE}$ is possible by perfusion scanning alone, without ventilation imaging.

\section{Pulmonary angiography}

Pulmonary angiography remains the standard of reference for detecting PE. It has proven to be highly accurate, but is nevertheless (because of its invasive nature) an underused test. With a 5-7 F catheter, the femoral vein is punctured and the catheter is advanced through the heart into the pulmonary trunk. After a trial injection of contrast, to look for possible central emboli, the catheter is placed in the right and left pulmonary artery. From each lung, angiographic series are made in two directions, posterior-anterior and oblique, preferably with the digital subtraction angiography technique. The criteria for acute PE are complete obstruction of a vessel or a filling defect (fig. 2) [36]. Complications of pulmonary angiography have decreased over the past years. Recent studies 
have shown mortality and morbidity rates of $0 \%$ and $0.3-1.8 \%$, respectively $[37,38]$. There are no absolute contraindications for pulmonary angiography, although several relative contraindications exist (such as contrast allergy, congestive heart failure, severe pulmonary hypertension and endocarditis).

Since angiography is the reference method, the sensitivity and specificity cannot be formally evaluated. The clinical validity was assessed in several studies [36, 39-41], which performed clinical followup, and revealed a sensitivity in the region of $98 \%$ and a specificity of $94-98 \%$. However, one should be aware of the fact that the reliability of pulmonary angiography decreases with diminishing calibre of the vessels, i.e. the interpretation becomes more difficult at a subsegmental level. Interobserver agreement for the diagnosis of clots beyond the segmental level is low; data of the PIOPED study showed an interobserver agreement of subsegmental PE of only $66 \%[5]$.

\section{Spiral computed tomography}

In 1978, SinNer [42] was the first to describe that pulmonary embolism could be directly depicted on conventional computed tomography (CT). The most reliable $\mathrm{CT}$ feature allowing definitive diagnosis of $\mathrm{PE}$ is the demonstration of an intravascular clot. This requires optimal enhancement of vessels to avoid pseudofilling defects in pulmonary arteries secondary to flow phenomena. With the use of dynamic scanning, emboli in central or lobar vessels could be detected [42-44]. However, conventional CT requires relatively long scan times, which allows respiratory motion artefacts that severely limit its usefulness as a diagnostic test for PE. Because of

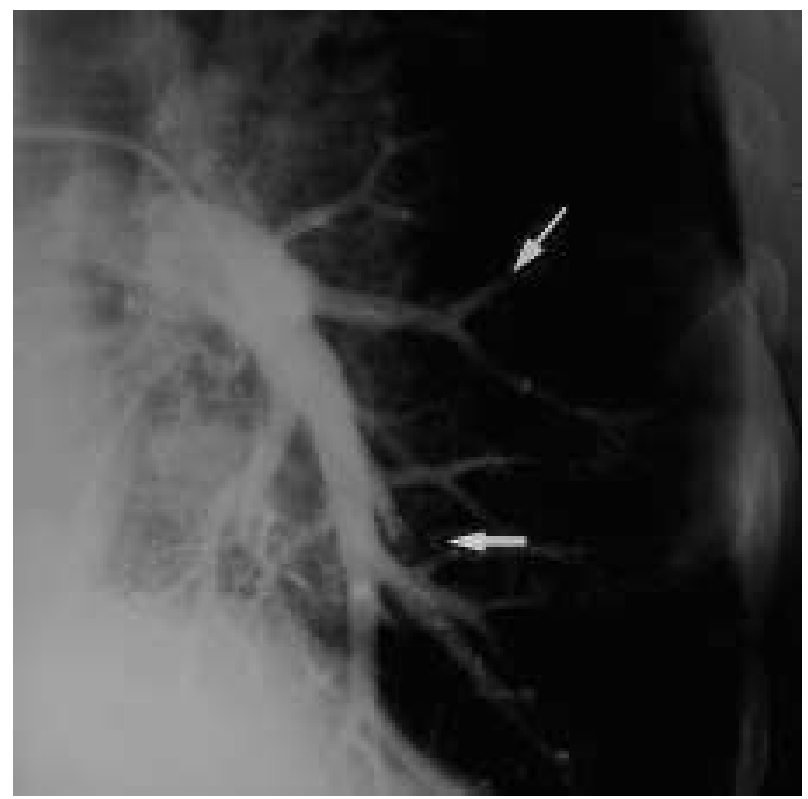

Fig. 2.-A pulmonary angiogram of the left lung, with filling defects in the lingula subsegmental artery and one of the basal lower lobe segmental arteries (arrows). recent improvements in $\mathrm{CT}$ technology, it is now possible to scan faster at the peak of contrast material opacification during a single breath-hold. With the use of spiral CT, continuous volume scanning is achieved; the patient is moved through the gantry while CT data are obtained [45]. PE can now be detected with spiral CT.

Clear demonstration of even one embolus is enough to make a definitive diagnosis. Arteries are considered thromboembolic if they show signs of a complete filling defect or cut off or a partial filling defect (fig. 3). Small (sub)segmental filling defects are called positive, only when a definite filling defect is seen on more than one axial image, in which artifactual causes have been excluded [46]. Secondary signs of PE include
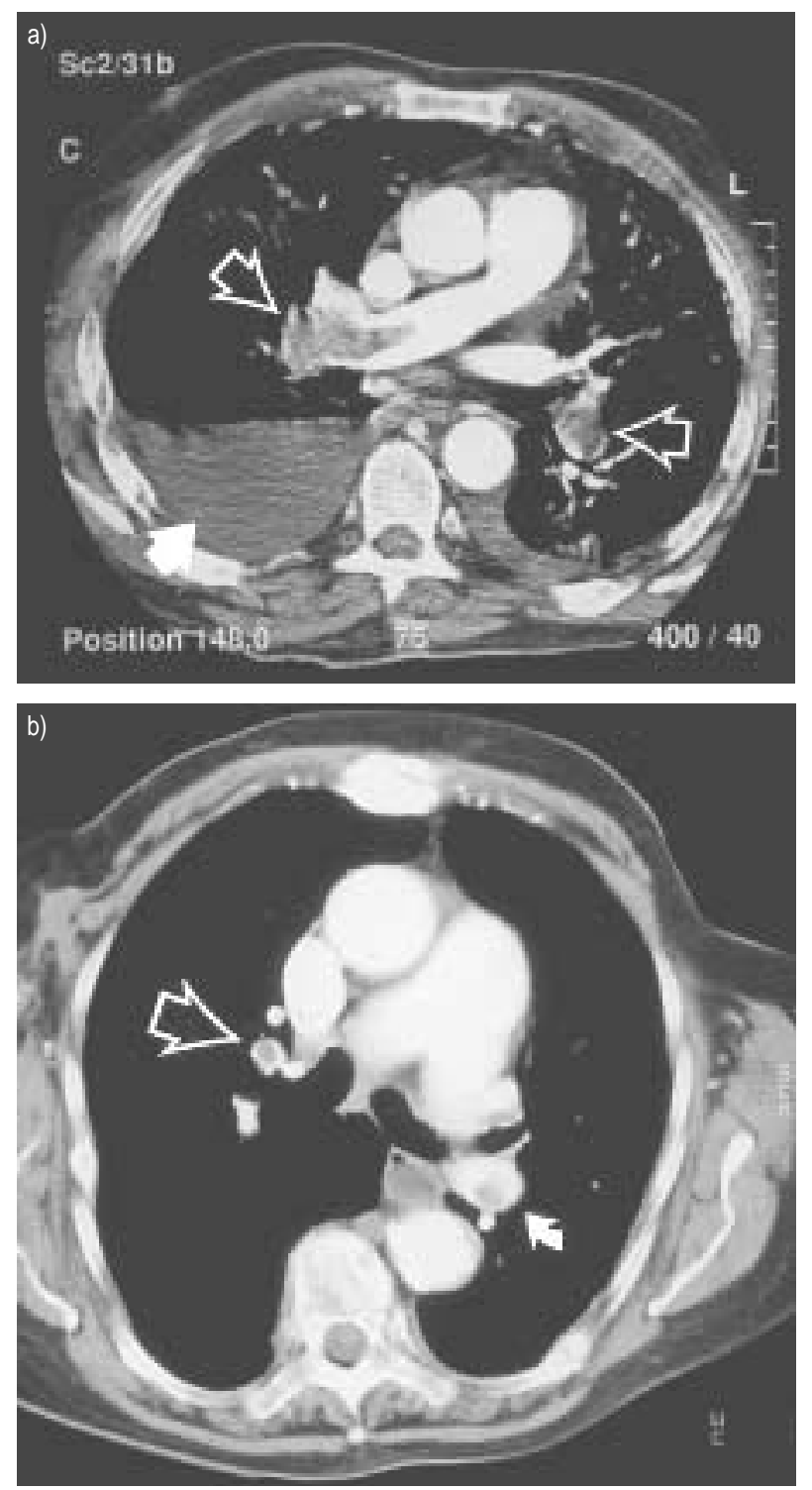

Fig. 3.-a) Spiral computed tomography (CT) at level of the right main pulmonary artery, a large clot is seen as a filling defect (open arrow) and also a clot in the left main lower lobe artery (open arrow). Note also, some pleural effusion on both sights (solid arrow). b) Another example of pulmonary emboli seen with Spiral CT. Emboli are seen as filling defects (arrows). 
Table 1.-Accuracy of spiral computed tomography for pulmonary embolism

\begin{tabular}{lccc}
\hline Author [ref] & Year & $\begin{array}{c}\text { Sensitivity } \\
\%\end{array}$ & $\begin{array}{c}\text { Specificity } \\
\%\end{array}$ \\
\hline REMY-JARDIN et al. [48] & 1992 & 100 & 96 \\
BLUM et al. [49] & 1994 & 100 & 100 \\
STEINER et al. [54] & 1994 & 100 & 100 \\
GOODMAN et al. [50] & 1995 & 63 & 89 \\
REMY-JARDIN et al. [52] & 1996 & 91 & 78 \\
VAN ROSSUM et al. [51] & 1996 & 88 & 94 \\
MAYO et al. [53] & 1997 & 87 & 95 \\
DRUCKER et al. [55] & 1998 & 60 & 81 \\
GRAG et al. [56] & 1998 & 57 & 100 \\
\hline
\end{tabular}

identification of a mosaic pattern caused by differential perfusion, infiltrates caused by haemorrhage, or infarction. However, these are all nonspecific signs and the diagnosis of PE cannot be made on these secondary signs only. Furthermore, acute large PE may cause dilatation of the right cardiac chambers, and finally, pleural effusion often accompanies acute $\mathrm{PE}$, but is nonspecific.

With this CT technique, good results have been shown (table 1) [47-55]. Multiple studies have demonstrated $\sim 90 \%$ sensitivity and specificity for PE [47-52]. In the first published studies in which the value of spiral CT for PE was evaluated, the figures of sensitivity and specificity were very high, even up to $100 \%$. But in these studies they mainly evaluated PE in central and segmental lobar arteries. The more recently published papers, where emboli in subsegmental arteries were also included, showed lower figures, with sensitivity ranging from $29-63 \%$ [49, 56]. In fact, currently, the main shortcoming of spiral CT for PE is the inability to show subsegmental emboli. But detection of PE in the subsegmental vessels can be improved by using a narrow collimation $(3 \mathrm{~mm}$ instead of the formerly often used $5 \mathrm{~mm}$ ) [57]. Improvement for evaluation of the subsegmental pulmonary arteries is particularly expected from the recently introduced multislice detector spiral CT, which seems very promising for CT angiography examinations.

Another question is, what is the prevalence of isolated subsegmental PE? There are no reliable data about the fraction of patients with PE limited to the subsegmental arteries only. The incidence of subsegmental PE has been reported in some studies, varying from $5-30 \%[5,49-51,58]$. Furthermore, the clinical importance of missing isolated subsegmental PE is currently unclear. Some investigators feel that small emboli must be considered the harbinger of future larger emboli and, therefore, clinically important. But other evidence suggests that small peripheral emboli might have little consequence for patients without underlying cardiopulmonary disease and no evidence of peripheral deep vein thrombosis [37, 59]. In a study by HuLL et al. [60], patients with nondiagnostic $V^{\prime} / Q^{\prime}$ lung scans, who had no substantial cardiac or pulmonary disease, received no anticoagulants unless there was evidence of deep vein thrombosis at impedance plethysmography. During follow-up these patients did very well; thromboembolic events recurred in $<3 \%$. In these patients with nondiagnostic $V^{\prime} / Q^{\prime}$ lung scans, small emboli must have gone undetected and untreated. However, in another study, done by FerRetTI et al. [61], who followed up patients with a negative spiral CT for PE, the recurrence rate was higher $(5.4 \%)$. In a study by GOTTSATER et al [62], who also followed up patients with a negative spiral CT for PE, the recurrence rate was only $1.4 \%$. So the reported figures are variable and the value of a negative spiral CT (and thus the chance of missing small subsegmental emboli) for PE is not clear yet. In addition, well-designed, large, prospective clinical outcome studies should still be performed to evaluate the relevance of negative spiral CT findings, especially with the presently available multiple slice CT technique.

Another advantage of spiral CT is the fact that, very often, an alternative diagnosis can be established $[61,63]$ which might explain patients' signs and symptoms. Only approximately one-third of patients suspected of PE will actually have PE, so most patients will have other causes for their complaints. CT will often allow an alternative diagnosis, because apart from the pulmonary arteries, lung parenchyma and mediastinal structures are also scanned. These alternative diagnoses could be, by example, pulmonary infiltrates, pleural effusion, tumour, aortic dissection, or bronchial or parenchymal diseases [61, 63]. However, it must always be remembered that these alternative diagnoses do not rule out PE. These other diagnoses could, of course, be coexistent or even represent secondary signs of PE, for example pulmonary infarction, presenting as a wedge-shaped pleural based infiltrate.

\section{Magnetic resonance imaging}

Conventional techniques in magnetic resonance imaging (MRI) are unable to distinguish segmental and subsegmental pulmonary vessels, because of the peculiar composition of lung tissue. New faster techniques, however, have made it possible to evaluate the pulmonary arteries. Numerous magnetic resonance angiography (MRA) techniques have been applied to study the pulmonary vasculature. The time-of-flight gradient-recalled-echo (GRE) technique is often used, where unexcited blood entering an image section appears bright compared with the surrounding tissue that has already been saturated by radio frequency pulses [64]. Good results have been achieved with this technique, but poor contrast resolution and signal-to-noise ratios still often make it difficult to distinguish thrombi from blood. The addition of contrast material makes the differentiation easier, increasing the sensitivity and specificity of MRA for PE. This regularly used technique is a contrast-enhanced three dimensional GRE MRA. Studies on this subject reported sensitivity rates of $71-87 \%$ and specificity rates of $95-97 \%$ [65-68].

A second method of imaging the pulmonary vessels is the use of MR perfusion imaging. After a bolus injection of a paramagnetic contrast agent, imaging of 
the lung is performed immediately and the entire lung perfusion can be mapped, including pulmonary vessels and parenchyma. In the case of PE, there will be a lack of perfusion in the specific area. This technique could also be combined with an MR ventilation technique, using aerosolized gadolinium. As a result MR-based $V^{\prime} / Q^{\prime}$ scans can be performed. The results of this technique have shown sensitivity figures $\sim 70 \%$ and specificity of $95-100 \%[66,69,70]$.

Although the new techniques of MRI seem very promising and accurate, the studies published on this subject report varying sensitivity and specificity with different techniques and expertise of the radiologist. Currently, MRI for the diagnosis of PE remains an experimental modality. Table 2 gives a summary of the performance of the various diagnostic tests for PE.

\section{Discussion}

The diagnosis of PE remains a vexing problem. The ideal diagnostic test should be accurate, safe, readily available and cost-effective. At present, none of the above mentioned tests meets all these criteria. It is, therefore, necessary to evaluate what combination of tests is most accurate. Recently, several studies have been published, in which different combinations of diagnostic tests (algorithms) for PE have been evaluated.

WeLLs et al. [71] evaluated the use of a clinical model for safe management of patients with suspected PE. In 1,239 patients the pretest probability for PE was categorized by a clinical model, $V^{\prime} / Q^{\prime}$ lung scans and bilateral leg ultrasonography were performed, and if necessary pulmonary angiography was performed. They found that three of the $665(0.5 \%)$ patients with low or moderate pretest probability and a nonhigh probability $V^{\prime} / Q^{\prime}$ lung scan, who were considered negative for PE, had PE or DVT during the 3-month follow-up. They concluded that management of patients with suspected $\mathrm{PE}$ on the basis of pretest probability and results of $V^{\prime} / Q^{\prime}$ lung scanning is safe.

PERRIER et al. [11] evaluated 444 outpatients clinically suspected of PE. In all patients, a rapid plasma D-dimer was measured. A normal D-dimer was used to rule out venous thromboembolism. Ultrasonography was performed in patients with a positive D-dimer test. If DVT was present, they were treated accordingly. In patients with a negative ultrasound, a $V^{\prime} / Q^{\prime}$ lung scan was then performed and only patients with a high clinical probability for

Table 2.-Accuracy of diagnostic tests for pulmonary embolism

\begin{tabular}{lcc}
\hline Test & $\begin{array}{c}\text { Sensitivity } \\
\%\end{array}$ & $\begin{array}{c}\text { Specificity } \\
\%\end{array}$ \\
\hline D-dimer & $90-99$ & $40-60$ \\
Ultrasound & 35 & 99 \\
Ventilation/perfusion lung scan & 98 & 10 \\
Spiral computed tomography & $57-100$ & $78-100$ \\
Magnetic resonance imaging & $71-87$ & $95-97$ \\
Pulmonary angiography & 98 & $94-98$ \\
\hline
\end{tabular}

PE and a nondiagnostic $V^{\prime} / Q^{\prime}$ lung scan underwent pulmonary angiography. Pulmonary angiography was done in 50 patients $(11 \%)$. With this study design, noninvasive diagnosis was possible in $394(89 \%)$ members of the entire study group. The negative predictive value of $\mathrm{D}$-dimer was $99.3 \%$. The 3-month thromboembolic risk in patients not given anticoagulants was $0.9 \%$. They concluded that a simple integrated diagnostic algorithm for suspected PE, combining clinical probability, a rapid plasma D-dimer ELISA, single lower limb venous ultrasonography and a $V^{\prime} / Q^{\prime}$ lung scan, allows a definitive noninvasive diagnosis in $89 \%$ of patients and appears to be safe. But it should be realized that these are the results of a study carried out in the outpatient clinic, and as was shown by Miron et al. [13], in contrast to outpatients, D-dimer measurements appear to be useless in hospitalized patients.

In a study by FERRETTI et al. [61], the role of spiral CT in 164 patients with nondiagnostic $V^{\prime} / Q^{\prime}$ lung scans and negative results from ultrasound of the legs was evaluated. When spiral CT demonstrated PE, anticoagulants where administrated. When spiral CT did not depict PE, anticoagulants were not started. Pulmonary angiography was performed only in those patients with high clinical suspicion for $\mathrm{PE}$ and negative spiral CT findings. Patients were followed for 3 months. At this follow-up, six of $112(5.4 \%)$ patients with negative spiral CT findings had experienced recurrent PE. They concluded that spiral CT allowed accurate diagnosis of acute PE in patients with nondiagnostic $V^{\prime} / Q^{\prime}$ lung scan and without DVT at ultrasound.

The issue of cost-effectiveness also needs to be addressed. While the effectiveness in making the diagnosis of $\mathrm{PE}$ is increasingly apparent, costing is open to a much wider interpretations. However, there are not many published studies that address this aspect. One is a cost-effective analysis study done by Hull et al. [27], in which three strategies were evaluated. The first consisted of a $V^{\prime} / Q^{\prime}$ lung scan followed by pulmonary angiography in cases of a nondiagnostic $V^{\prime} / Q^{\prime}$ lung scan. The second strategy consisted of a $V^{\prime} / Q^{\prime}$ lung scan, followed by a single noninvasive leg test and pulmonary angiography as final test. In the third strategy, serial leg tests were used instead of a single test. The authors concluded that the strategy that requires pulmonary angiography in the fewest patients is a combination of $V^{\prime} / Q^{\prime}$ lung scans and serial noninvasive leg tests. This strategy also proved to be the most cost-effective.

Another study was performed by VAN ERKEL et al., [72] with an updated analysis in 1998 [73]. In their analysis, 16 realistic combinations of several diagnostic tests (including $V^{\prime} / Q^{\prime}$ lung scan, leg ultrasound, D-dimer, spiral CT and pulmonary angiography) were evaluated for patients with and without comorbidity. The authors found that strategies using spiral CT had lower cost (with the best strategy being ultrasound followed by spiral CT) and higher survival than strategies that use pulmonary angiography. Comparison of patients with and without comorbidity demonstrated that strategies including D-dimer tests are most costly in patients with comorbidity. 
Obviously, comorbidity leads to less favourable costeffectiveness of $\mathrm{D}$-dimer strategies. The authors also concluded that there is a prominent role for spiral CT in the diagnosis of PE. However, it should not be forgotten that this study uses theoretical recommendations for an optimally cost-effective algorithm and can therefore serve as best approximations only.

In another study, a total of 41 diagnostic strategies were evaluated using a mathematical model [74]. Several assumptions were made to build a model for cost-effective analysis. These related to the prevalence of $\mathrm{PE}$ in the population under investigation, the influence of therapeutic interventions on the natural course of PE, the adverse events due to therapeutic interventions, the performance of the diagnostic tests and the overall costs. The studied strategies included $V^{\prime} / Q^{\prime}$ lung scanning, ultrasonography of the legs, pulmonary angiography, spiral CT and D-dimer test, all combined in different combinations. The primary outcome measure for each strategy was the average mortality and cost per patient, defined as the excess mortality and cost compared to the optimal strategy (the strategy that assumes that the clinician is always correct in the diagnosis and treatment). A costeffectiveness analysis was performed in which, for each strategy, the costs per life saved were calculated compared to the natural course of PE. They found that the mortality was lowest in all strategies that used pulmonary angiography as a final diagnostic test. The strategy that consisted of a $V^{\prime} / Q^{\prime}$ lung scan followed by ultrasound of the legs and finally, if necessary in cases of negative findings, pulmonary angiography, had the lowest mortality and costs. The performance of spiral CT in a strategy prior to pulmonary angiography (apart from the aforementioned strategy) generally lead to a reduction in mortality. Strategies that incorporated $\mathrm{D}$-dimer tests resulted in $\mathrm{a} \geqslant 2$-fold increase in mortality when compared to other strategies using pulmonary angiography. The cost of noninvasive strategies was much lower than those containing pulmonary angiography, but the mortality is deemed unacceptably high. They found that pulmonary angiography is still required as a final diagnostic step in any approach but it seems worthwhile to reduce the number of patients who require pulmonary angiography by prior performance of noninvasive diagnostic tests. It was concluded that the strategy which incorporates a $V^{\prime} / Q^{\prime}$ lung scan, followed by ultrasonography of the legs and finally pulmonary angiography, is still the strategy of choice. However, no major difference in outcome exists between the strategy where spiral CT is used after a perfusion lung scan, then followed by a final pulmonary angiography. This strategy will result in a $16 \%$ reduction in angiographic procedures, and in this strategy, the ventilation lung scan is replaced by spiral CT. Since spiral CT is more widely available as a routine test than a ventilation lung scan, it seemed logical to switch over to spiral CT.

In summary, several results of different diagnostic algorithms, evaluated in several studies are variable but acceptable and the choice for which strategy to use is highly dependent on the availability of the diagnostic tests within a local hospital. In the author's opinion, the perfusion lung scan remains a key factor, since a normal perfusion lung scan is highly accurate for exclusion of pulmonary embolism in a considerable number of patients and makes further testing for many patients redundant. After the perfusion lung scan, spiral computed tomography can be performed in cases of an abnormal perfusion lung scan, followed by a pulmonary angiography in patients with a negative spiral computed tomographic scan and high clinical suspicion of pulmonary embolism. Although the willingness of the clinician to perform pulmonary angiography is still restricted, pulmonary angiography is required as the final step in any approach to exclude or detect pulmonary embolism.

\section{References}

1. Gjores JE. The incidence of venous thrombosis and its sequelae in certain districts of Sweden. Acta Chir Scand 1965; Suppl. 206: 1-88.

2. Anderson Jr FA, Wheeler HB, Goldberg RJ, et al. A population-based perspective of the hospital incidence and case-fatality rates of deep venous thrombosis and pulmonary embolism. Arch Int Med 1991; 151: 933938.

3. Robin ED. Overdiagnosis and overtreatment of pulmonary embolism: the emperor may have no clothes. Ann Int Med 1977; 87: 775-781.

4. Hull RD, Hirsh J, Carter CJ, et al. Diagnostic value of ventilation-perfusion lung scanning in patients with suspected of pulmonary embolism. Chest 1985; 88: 819-828.

5. PIOPED investigators. Value of ventilation-perfusion scan in acute pulmonary embolism: results of the prospective investigation of pulmonary embolism diagnosis. JAMA 1990; 263: 2753-2759.

6. Egermayer P, Town GI. The clinical significance of pulmonary embolism: uncertainties and implications for treatment - a debate. J Intern Med 1997; 241: 5-10.

7. Miniati M, Prediletto R, Formichi B, et al. Accuracy of clinical assessment in the diagnosis of pulmonary embolism. Am J Respir Crit Care Med 1999; 159: 864 871.

8. Ginsberg JS, Brill-Edwards PA, Demers C, Donovan D, Panju A. D-dimer in patients with clinically suspected pulmonary embolism. Chest 1993; 104: 1679-1684.

9. Van Beek EJR, van den Ende B, Berckmans RJ, et al. A comparative analysis of $\mathrm{D}$-dimer assays in patients with clinically suspected pulmonary embolism. Thromb Haemostas 1993; 70: 408-413.

10. Jansen $\mathrm{MCH}$, Heebels $\mathrm{AE}$, de Metz $\mathrm{M}$, et al. Reliability of five rapid D-dimer assays compared to ELISA in the exclusion of deep venous thrombosis. Thromb Haemostas 1997; 77: 262-266.

11. De Moerloose P, Desmarais S, Bounameaux $H$ et al. Contribution of a new, rapid, individual, and quantitative automated D-dimer ELISA to exclude pulmonary embolism. Thromb Heamostas 1996; 75: 11-13.

12. Perrier A, Desmarais S, Miron MJ, et al. Non-invasive diagnosis of venous thromboembolism in outpatients. Lancet 1999; 353: 190-195.

13. Miron MJ, Perrier A, Bounameaux H, et al. Contribution of non-invasive evaluation to the diagnosis 
of pulmonary embolism in hospitalised patients. Eur Respir J 1999; 13: 1365-1370.

14. Becker DM, Philbrick JT, Abbitt PL. Real-time ultrasonography for the diagnosis of lower extremity deep venous thrombosis: the wave of the future? Arch Int Med 1989; 149: 1731-1734.

15. Lensing AWA, Prandoni P, Brandjes D, et al. Detection of deep venous thrombosis by real-time B-mode ultrasonography. $N$ Eng J Med 1989; 320: 342-345.

16. Cogo A, Lensing AWA, Koopman MMW, et al. Compression ultrasonography for the diagnosis management of patients with clinically suspected deep vein thrombosis: prospective cohort study. BMJ 1998; 316 : 17-20.

17. Heijboer H, Buller HR, Lensing AWA, et al. A comparison of real-time compression ultrasonography with impedance plethysmography for the diagnosis of deep-vein thrombosis in symptomatic outpatients. $N$ Eng J Med 1993; 329: 1365-1369.

18. Hull RD, Raskob GE, Ginsberg JS, et al. A noninvasive strategy for the treatment of patients with suspected pulmonary embolism. Arch Int Med 1994; 154: 289-297.

19. Schiff MJ, Feinberg AW, Naidich JB. Non-invasive venous examinations as a screening test for pulmonary embolism. Arch Int Med 1987; 147: 505-507.

20. Killewich LA, Nunnelee JD, Auer AL. Value of lower extremity venous duplex examination in the diagnosis of pulmonary embolism. J Vasc Surg 1993; 17: 934 939.

21. Quinn RJ, Nour R, Butler SP, et al. Pulmonary embolism in patients with indeterminate probability lung scans: diagnosis with doppler venous US and D-dimer measurements. Radiology 1994; 190: 509-511.

22. Smith LL, Iber C, Sirr S. Pulmonary embolism: confirmation with venous duplex US as adjunct to lung scanning. Radiology 1994; 191: 143-147.

23. Christiansen F, Kellerth B, Anderson T, Ragnarson A, Hjortevang F. Ultrasound at scintigraphic intermediate probability of pulmonary embolism. Acta Radiol 1996; 37: 14-17.

24. Turkstra F, Kuijer PMM, van Beek EJR, et al. Value of compression ultrasonography for the detection of deep venous thrombosis in patients suspected of having pulmonary embolism. Ann Int Med 1997; 126: $775-781$.

25. Stein PD, Russsel D, Hull RD, Saltzman HA, Pineo G. Strategy for diagnosis of patients with suspected acute pulmonary embolism. Chest 1993; 103: 1553-1559.

26. Oudkerk M, van Beek EJR, van Putten WLJ, Buller HR. Cost-effectiveness analysis of various strategies in the diagnostic management of pulmonary embolism. Arch Int Med 1993; 153: 947-954.

27. Hull RD, Feldstein W, Stein PD, Pineo GF. Costeffectiveness of pulmonary embolism diagnosis. Arch Int Med 1996; 156: 68-72.

28. Beecham RP, Dorfman GS, Spearman MP, et al. Is bilateral lower extremity compression sonography useful and cost-effective in the evaluation of suspected pulmonary embolism? AJR 1993; 161: 1289-1292.

29. Van Rossum AB, van Houwelingen HC, Kieft GJ, Pattynama PMT. Prevalence of deep vein thrombosis in suspected and proven pulmonary embolism: a metaanalysis. Br J Radiol 1998; 71: 1260-1264.

30. McNiel BJ, Holman BL, Adelstein SJ. The scintigraphic definition of pulmonary embolism. JAMA 1974; 227: 753-756.
31. Biello DR, Mattar A, McKnight RC, Siegel BA. Ventilation-perfusion studies in suspected pulmonary embolism. AJR 1979; 133: 1033-1037.

32. Hull RD, Raskob GE, Hirsh J. Low-probability lung scans: a need for change. Ann Int Med 1991; 114: 142143.

33. Van Beek EJR, Tiel-van Buul MMC, Buller HR, et al. The value of lung scintigraphy in the diagnosis of pulmonary embolism. Eur J Nucl Med 1993; 20: 173181.

34. Hull RD, Raskob GE, Hirsh J. Clinical validity of a normal perfusion lung scan in patients with suspected pulmonary embolism. Chest 1990; 97: 23-26.

35. Miniati M, Pistolesi M, Marini C, et al. Value of perfusion lung scan in the diagnosis of pulmonary embolism: results of the prospective investigative study of acute pulmonary embolism diagnosis (PISA-PED). Am J Resp Crit Care Med 1996; 154: 1387-1393.

36. Stein PD, Athanasoulis C, Alavi A, et al. Complications and validity of pulmonary angiography in acute pulmonary embolism. Circulation 1992; 85: 462-468.

37. Hudson ER, Smith TP, McDermott VG, et al. Pulmonary angiography with iopamidol: complications in 1434 patients. Radiology 1996; 198: 61-65.

38. Van Rooij WJ, den Heeten GJ, Sluzewski M. Pulmonary embolism: diagnosis in 211 patients with the use of selective pulmonary digital substraction angiography with a flow-directed catheter. Radiology 1995; 195: 793-797.

39. Van Beek EJR, Reekers JA, Batchelor D, et al. Feasibility, safety and clinical utility of angiography in patients with suspected pulmonary embolism and nondiagnostic lung scan findings. Eur Radiol 1996; 6: 415419.

40. Johnson MS, Stine SB, Shah $\mathrm{H}$, et al. Possible pulmonary embolus: evaluation with digital subtraction versus cut-film angiography - prospective study in 80 patients. Radiology 1998; 207: 131-138.

41. Hagspiel KD, Polak JF, Grassi CJ, et al. Pulmonary embolism: comparison of cut-film and digital pulmonary angiography. Radiology 1998; 207: 139-145.

42. Sinner WN. Computed tomographic patterns of pulmonary thromboembolism and infarction. $J$ Comput Assist Tomogr 1978; 2: 395-399.

43. Godwin JD, Webb WR, Gamsu G, Overfors CO. Computed tomography of pulmonary embolism. AJR 1980; 135: 691-695.

44. Kalebo P, Wallin J. Computed tomography in massive pulmonary embolism. Acta Radiol 1989; 30: 105-107.

45. Kalender WA, Sieber W, Klotz E, Vock P. Spiral volumetric CT with single breathhold technique, continuous transport and continuous scanner rotation. Radiology 1990; 176: 181-183.

46. Kuzo RS, Goodman LR. CT evaluation of pulmonary embolism: technique and interpretation. AJR 1997; 169: 959-965.

47. Remy-Jardin M, Remy J, Wattine L, Giraud F. Central pulmonary thromboembolism diagnosis with spiral volumetric CT with the single breath hold technique: comparison with pulmonary angiography. Radiology 1992; 185: 839-845.

48. Blum AG, Delfau F, Grignon B, et al. Spiral computed tomography versus pulmonary angiography in the diagnosis of acute massive pulmonary embolism. Am J Cardiol 1994; 74: 96-98.

49. Goodman LR, Curtin JJ, Mewissen MW, et al. 
Detection of pulmonary embolism in patients with unresolved clinical and scintigraphic diagnosis: helical CT versus angiography. AJR 1995; 164: 1369-1374

50. Van Rossum AB, Pattynama PMT, Tjin A, Ton ER, et al. Pulmonary embolism: validation of spiral CT angiography in 149 patients. Radiology 1996; 201: $467-470$.

51. Remy-Jardin $\mathrm{M}$, Remy $\mathrm{R}$, Deschildre $\mathrm{F}$, et al. Diagnosis of pulmonary embolism with spiral CT: comparison with pulmonary angiography and scintigraphy. Radiology 1996; 200: 699-706.

52. Mayo JR, Remy-Jardin M, Muller NL, et al. Pulmonary embolism: prospective comparison of spiral CT with ventilation-perfusion scintigraphy. Radiology 1997; 205: 447-452.

53. Steiner $\mathrm{P}$ von, Phillips F, Wesner $\mathrm{D}$, et al. Primar diagnostik und Verlaufskontrolle der akuten lungenembolie: Vergleich zwischen digitaler substractionangiographie und spiral CT. Fortschr Rontgenstr 1994; 161: 285-291.

54. Drucker EA, Rivitz SM, Shepard JAO, et al. Acute pulmonary embolism: assessment of helical CT diagnosis. Radiology 1998; 209: 235-241.

55. Grag K, Welsh CH, Feyerbend AJ, et al. Pulmonary embolism: diagnosis with spiral CT and ventilationperfusion scanning - correlation with pulmonary angiographic results or clinical outcome. Radiology 1998; 208: 201-208.

56. Mullins MD, Becker DM, Hagspiel KD, Philbrick JT. The role of spiral volumetric computed tomography in the diagnosis of pulmonary embolism. Arch Int Med 2000; 160: 293-298.

57. Remy-Jardin M, Remy J, Artaud D, Deschildre F, Duhamel A. Peripheral pulmonary arteries: optimatization of the spiral CT acquisition protocol. Radiology 1996; 207: 157-163.

58. de Monye W, van Strijen MJL, Huisman MV, Kieft GJ, Pattynama PMT. Suspected pulmonary embolism: prevalence and anatomic distribution in 487 consecutive patients. Radiology 2000; 215: 184-188.

59. Drofman GS, Cronan JJ, Truper TB, et al. Occult pulmonary embolism: a common occurence in deep vein thrombosis. AJR 1987; 148: 263-266.

60. Hull RD, Raskob GE, Coates G, Panju AA, Gill GJ. A new non-invasive management for patients with suspected pulmonary embolism. Arch Int Med 1989; 149: 2549-2555.

61. Ferretti GR, Bosson JL, Buffaz PD, et al. Acute pulmonary embolism: role of helical CT in 164 patients with intermediate probability at ventilationperfusion scintigraphy and normal results at duplex US of the legs. Radiology 1997; 205: 453-458.

62. Gottsater A, Berg A, Centergard J, et al. Clinically suspected pulmonary embolism: is it safe to withhold anticoagulation after a negative spiral CT? Eur Radiol 2001; 11: 65-72.

63. Van Rossum AB, Pattynama PMT, Mallens WMC, Hermans J, Heijerman HGM Can helical CT replace scintigraphy in the diagnostic process in suspected pulmonary embolism? A retrospective-prospective cohort study focusing on total diagnositic yield. Eur Radiol 1998; 8: 90-96.

64. Mitchell DG. MRI Principles. Philadelphia, PA, W.B. Saunders Co., 1999.

65. Isoda $\mathrm{H}$, Ushimi $\mathrm{T}$, Masui $\mathrm{T}$, et al. Clinical evaluation of pulmonary 3D time-of-flight MRA with breathholding using contrast media. J Compt Assist Tomogr 1995; 19: 911-919.

66. Meaney JF, Weg JG, Chenevert TL, et al. Diagnosis of pulmonary embolism with magnetic resonance angiography. New Eng J Med 1997; 336: 1422-1427.

67. Wielopolski PA, Oudkerk M, Hicks SG, Berghout A. Breath-hold 3D MR pulmonary angiography after contrast administration in patients with pulmonary embolism: correlation with conventional pulmonary angiography. Radiology 1996; 201: 202.

68. Oudkerk M, de Bruin HG, Wielopolski PA, et al. Pulmonary embolism detection using contrastenhanced, breath-hold 3D magnetic resonance angiography. In: Oudkerk M, Edelman RR, eds. Highpower gradient MR imaging. Advances in MRI II. Berlin, Blackwell Science Publishers, 1997: 78-86.

69. Loubeyre P, Revel D, Douek P, et al. Dynamic contrast enhanced MR angiography of pulmonary embolism: comparison with pulmonary angiography. Am J Roeng 1994; 162: 1035-1039.

70. Amundsen T, Kvaerness J, Jones R, et al. Pulmonary embolism: detection with MR perfusion imaging of lung - feasibility study. Radiology 1997; 203: 181-185.

71. Wells PS, Ginsberg JS, Anderson DR, et al. Use of a clinical model for safe management of patients with suspected pulmonary embolism. Ann Int Med 1998; 129: 997-1005.

72. Van Erkel AR, van Rossum AB, Bloem JL, Kievit J, Pattynama PMT. Spiral CT angiography for suspected pulmonary embolism: a cost-effectiveness analysis. Radiology 1996; 201: 29-36.

73. Van Erkel AR, Pattynama PMT. Cost-effective diagnostic algorithms in pulmonary embolism: an updated analysis. Acad Radiol 1998; 5: Suppl. 2, S321S327.

74. Oudkerk M, van Beek EJR, van Putten WLJ, Rutten FFH. Management strategies in patients with suspected pulmonary embolism. In: Oudkerk $\mathrm{M}$, van Beek EJR, ten Cate JW, eds. Pulmonary embolism. Blackwell Science, 1999. 